\title{
NUMI PROTON KICKER EXTRACTION SYSTEM *
}

\author{
C. C. Jensen, G. A. Krafczyk, Fermilab, Batavia IL 60510, USA
}

\section{Abstract}

This system extracts up to $9.6 \mu$ s of $120 \mathrm{GeV}$ beam every 1.87 seconds for the NuMI beamline neutrino experiments. A pulse forming network consisting of two continuous wound coils and 68 capacitors was designed and built to drive three kicker magnets. The field stability requirement is better than $\pm 1 \%$ with a field rise time of $1.52 \mu \mathrm{s}$. New kicker magnets were built based on the successful traveling wave magnets built for the Main Injector. Two of these magnets are in series which places a serious constraint on the rise time of the pulser. A forced cooling system using Fluorinert ${ }^{\circledR}$ was designed for the magnet termination resistors to maintain the field flatness and amplitude stability.

\section{INTRODUCTION}

A fast single turn extraction kicker was required for the NuMI project to spill $120 \mathrm{GeV}$ protons onto a target. The extraction kicker has to rise in the $\sim 1.5 \mu$ s abort gap of the Fermilab Main Injector and then extract the following $9.6 \mu \mathrm{s}$ ( 6 batches) of beam down the beam line. There is a second operational mode that will initially be more common. In this "mixed mode", $1.6 \mu \mathrm{s}$ of beam (a single batch) is destined for antiproton production and only 8.0 $\mu \mathrm{s}$ of beam (5 batches) is extracted to NuMI. The extraction angle was required to be very stable pulse to pulse and have minimal ripple. The final requirements are shown in Table I. They changed over the design stage of the project. The kick angle increased by more than $60 \%$ requiring an additional magnet while the rise time could not be increased. There was a desire to do better than $\pm 0.5 \%$ in flat top ripple and better than $\pm 0.5 \%$ in pulse to pulse repeatability. Initial tests indicate that the desired goals are met for this kicker for mixed mode.

Table I. Specifications

\begin{tabular}{|l|l|}
\hline Total Integrated Field & $3.6 \mathrm{kG} \mathrm{m}(\mathrm{nom})$ \\
\hline Field Rise Time & $1.52 \mu \mathrm{s}(\max )$ \\
\hline Flat Top Length & $9.6 \mu \mathrm{s}(\mathrm{min})$ \\
\hline Flat Top ripple & $\pm 1 \%$ \\
\hline Pulse to Pulse Repeatability & $\pm 0.5 \%$ over 8 hours \\
\hline Magnet Aperture & $2 \mathrm{~m} \times 10.7 \mathrm{~cm} \times 5.2 \mathrm{~cm}$ \\
\hline Repetition Period & 1.87 seconds $(\mathrm{min})$ \\
\hline
\end{tabular}

\section{POWER SUPPLY}

\section{Power Forming Network}

A pulse forming network was previously used for proton extraction to Tevatron for fixed target experiments at a repetition rate of 1 per 30 seconds. This PFN design was studied and the actual components reviewed for issues of suitability. There were several shortcomings of

* Fermilab is operated by Universities Research Association, Inc. under contract DE-AC02-76CH03000 with the U.S. Department of Energy this design. There were many high current connections, each cell inductor was individually wound, and the connections showed signs of arcing. The coupling from cell to cell was less than $1 \%$ which caused poor flattop performance.

The LHC injection kicker system had recently been modeled and prototyped by Wait et al. at TRIUMF [1]. This scheme uses a machined coil form and a continuously wound coil to give the desired coupling and a more consistent inductance. In addition, the capacitor connections do not conduct the full rms current. Only the capacitor current for each section passes through a bolted connection. Two PFNs in parallel are used to realize a pulse that is flatter than a single PFN. This is a result of ordering of the capacitors to match low and high values in pairs and match the propagation time at each section along the length of the PFN.

This LHC injection pulser design was adopted with several changes. First, the taps in the coil were soldered in place to reduce complexity and increase reliability. Second, the damping resistors across each PFN cell were dropped. The purpose of these resistors was to dampen flattop oscillation, but we used a different technique for the Fermilab design. The ringing period of the PFN cell was picked to be the kicker magnet fill time. Since the magnet integrates the voltage between terminals, a more uniform magnetic field is obtained if the voltage transit time and the ringing period are the same. The design tradeoff however is an increase in the number of cells to accomplish the required period. Finally, since the NuMI kicker is an extraction system, there was no dump / clip switch as there is no fall time requirement.

The original benefit from the increase in the number of PFN cells was to provide margin in the field rise time. This proved serendipitous when the total kick strength was increased. All the available rise time was used up by adding a second magnet in series with one of the original two, the $2+1$ solution. This choice was a compromise between cost and performance. The existing spare magnet and previous magnet design work could be used in this $2+1$ configuration. However, the field rise time was increased over a new, shorter, $2+2$ magnet design. Beam line space was also at a premium and the $2+2$ magnet design would have required more space.

One final issue appeared during full voltage testing. The coupling from cell to cell was determined from measurements to be $17 \%$ instead of the design value of $13 \%$ for best flatness. Recall that the equivalent circuit of positive coupling is negative inductance in series with the capacitor. The result is a flatter or slightly increasing phase velocity as a function of frequency in the network. A coupling of larger than $8 \%$, for an ideal line, gives a 
rising current tail at the end of the pulse. The larger design coupling, beyond the $8 \%$ value, was used to compensate for the equivalent series inductance of the capacitor and connections. The prototype used a low voltage mica capacitor and the final capacitors have only $20 \mathrm{nH}$ of ESL. However, the connection loop inductance of the final system must be much lower than the prototype as the effective coupling is $15 \%$ instead of $8 \%$. The higher coupling could have been reduced after these measurements but it has the beneficial effect of further flattening the pulse. Since there is no fall time requirement for this system, we chose not to change the coupling.

Figure 1 shows the pulse response of the PFN into a 5 Ohm resistive load at nominal current, $5 \mathrm{kA}$. Figure 2 shows a cross section of half the final dual PFN tank assembly. The other half is a mirror image. There is sufficient space to replace a failed capacitor by pulling it out to the side. The entire tank is filled with mineral oil.

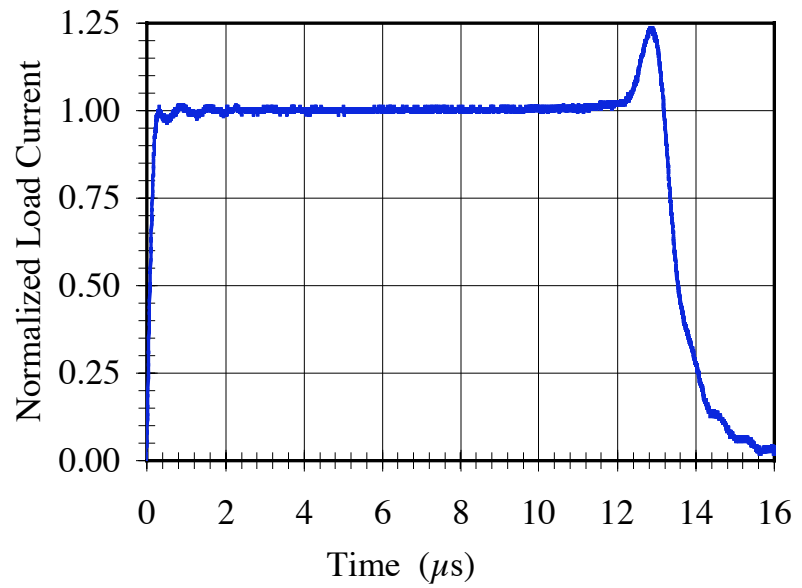

Figure 1

Normalized Load Current ( 5 kA) into Resistive Load

\section{Thyratron}

The existing $5 \mathrm{Ohm}$ system for Main Injector uses an E2V CX1592C hydrogen thyratron. This tube has shown good life in that system and using the same thyratron would reduce the number of spare thyratron types required. The thyratron housing is low inductance with a coaxial shield around the thyratron. The total inductance measured from PFN to output cable, with a $8 \mathrm{~cm}$ rod in place of the thyratron, is $200 \mathrm{nH}$. This inductance is not required to be very small, but should not be much larger. The switch housing inductance is added to the first cell of the PFN, but since it has no coupling to the adjacent cell a larger value degrades performance.

\section{Charging Supply}

The charging supply was made to specification. There are very few companies that make a capacitor charging supply at the $60 \mathrm{kV}$ level and the cost per watt is at least $70 \%$ higher than for a $40 \mathrm{kV}$ output. A resonant charging system consisting of a $5 \mathrm{kV}$ supply, a pulse transformer and a thyristor switch was investigated. It was rejected because the cost would have been approximately double that of the supply finally chosen. The supply was purchased from General Atomics Energy Products and is a $60 \mathrm{kV}, 80 \mathrm{~mA}, 5 \mathrm{~kJ} / \mathrm{sec}$ peak, $2.5 \mathrm{~kW}$ supply.

The repetition rate for this supply is several seconds. Fermilab experience is that this is the most troublesome cycle time for long operations life. The semiconductors have enough time to come to full operating temperature and then cool off each cycle. Because we required a semiconductor temperature rise of $<30 \mathrm{C}$, this led the manufacturer to use higher power devices to meet our need. The supply has worked for several months. There were some initial concerns regarding the output cable termination at the supply. This connector might have some long term high voltage reliability issues as there is some acoustic noise when running at high voltage.

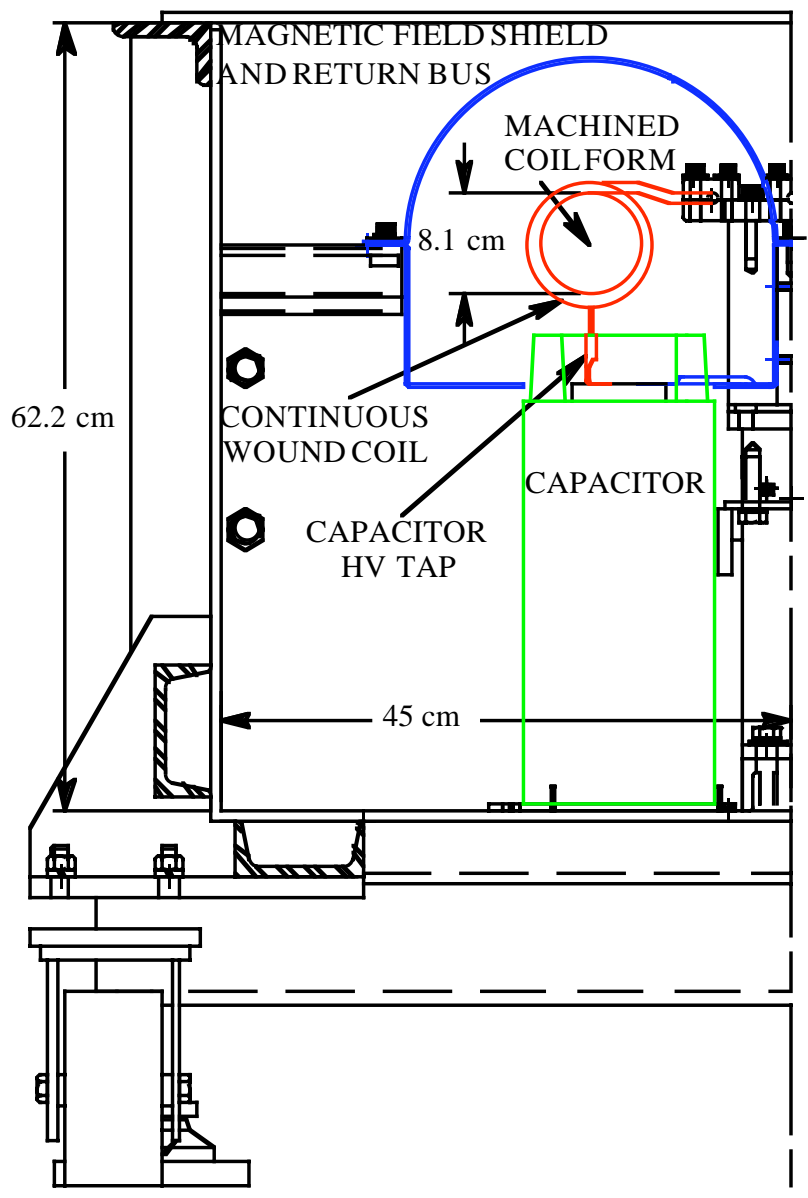

Figure 2

Half of PFN Tank showing one coil and capacitor

\section{MAGNET}

Traveling wave magnets were designed for $150 \mathrm{GeV}$ proton extraction when the Main Injector was built. These magnets have operated for over five years at $120 \mathrm{GeV}$ and one pulse per 2.4 seconds for antiproton production and have a magnetic field fill time of $550 \mathrm{~ns}$. 
Most kicker magnets at Fermilab are built around a ceramic vacuum chamber. Getting these chambers is the key to success. Unfortunately, there were problems mid way through the project. An existing chamber was broken during the vacuum flanging process and the company that had made them in the past changed business focus. We were forced to recycle chambers from old magnets and to slightly modify the magnet design. We have recently had several chambers made at 93" long after a two year development effort with a different vendor.

The original magnet design consisted of 92 sections each of ferrite and capacitor (see Figure 3) for a total magnetic length of $2 \mathrm{~m}$. This number of sections did not increase the cutoff frequency of the magnet however, due to a large structure ESL in the capacitor connections. The magnet parameters were measured and several attempts were taken to reduce this ESL. Finally, the magnet was modeled and it was found that 46 sections, with the same total inductance and capacitance, would give a response equivalent to the existing magnet and be $\sim 10 \%$ less expensive to manufacture.

The key to high performance at reduced cost in this magnet is an adjustable gap width. High precision capacitor values are expensive to purchase, but groups of capacitors with tight relative values are more cost effective. This tight grouping in capacitance value can be used by adjusting the magnetic width to meet the tight impedance requirements $( \pm 1 \%)$. This technique was used most recently on the Tevatron injection kickers [2].

The magnet load must also be closely matched to the magnet impedance. To get the best performance in the fastest kicker systems we have mounted the load directly on the magnet. The NuMI system also required an actively cooled load to maintain a constant resistance value [3]. This is needed for both pulse to pulse variation and flat top variation.

\section{RESULTS}

The system was measured during commissioning. The standard way we determine fast pulsed magnetic fields is to measure the magnet input and output voltage, subtract the two and integrate. This magnet does not have any voltage monitors, so the voltage across the load current viewing resistor was used as the output. The only signal available at the magnet input is the PFN output current transformer. Because the two monitors have different characteristics, it was difficult to determine the accuracy of the calculation of the field from measurements. Spice models of the PFN and of the magnet were verified with measurements at low voltage to within several percent.

The response was measured with a high precision beam position monitoring system designed at Fermilab for the NuMI beamline. This system has a stability of several $\mu \mathrm{m}$ over several hours. The flatness measured over many pulses at several points is shown in Figure 4 with the load temperature stable within $\pm 1 / 4 \mathrm{C}$. This is a measurement of orbit change in the Main Injector between the kicker and extraction Lambertson after the kicker is fired.

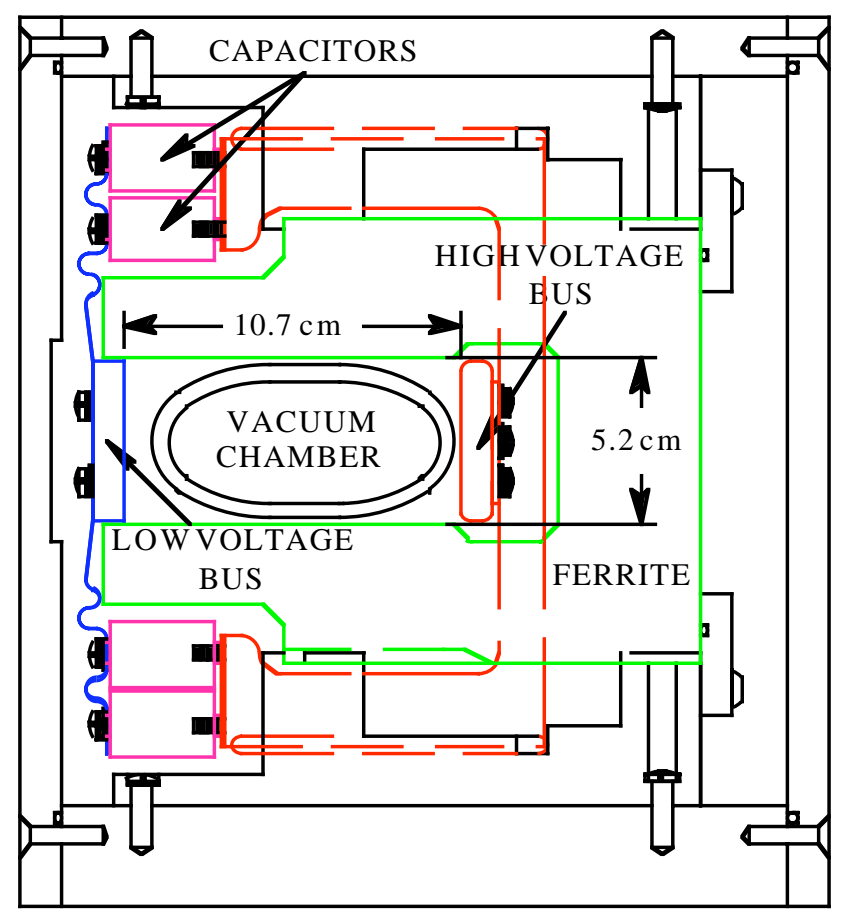

Figure 3 Cross Section of Kicker Magnet

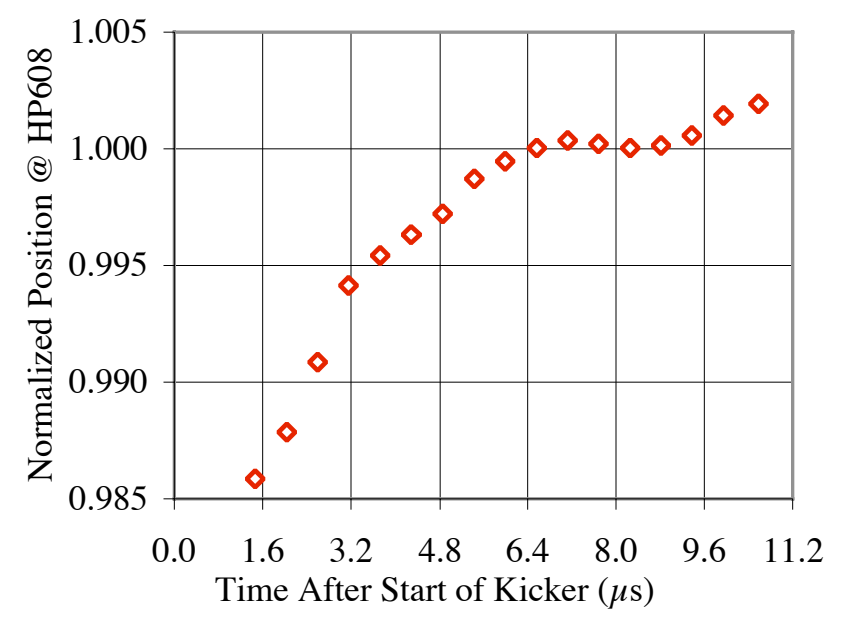

Figure 4 Normalized Beam Position Change (41.8 mm)

\section{ACKNOWLEDGEMENTS}

Thanks to Alberto Marchionni, Dharmaraj Indurthy, Phil Adamson; Qiang Du; Ken Kellogg, Dirong Chen and Cliff Foster for beam measurement, testing and assembly.

\section{REFERENCES}

[1] G.D. Wait, M.J. Barnes, E. Carlier. L. Ducimetiere, G.H. Schroeder, E.B. Vossenberg, "High Voltage Measurements on a Prototype PFN for the LHC Injection Kickers", PAC 1999, pp1509-1511.

[2] C.C. Jensen, "A Fast Injection Kicker Magnet for the Tevatron", PAC 2001, pp 3720-2723.

[3] S. Reeves, C. C. Jensen, "Numi Proton Kicker Extraction Magnet Termination Resistor System", PAC 2005. 